\title{
The temporal order of fluctuations in atopic disease symptoms and attention-deficit/hyperactivity disorder symptoms: a time-series study in ADHD patients
}

\author{
Jurjen van der Schans ${ }^{1}$ (D - Qi Cao ${ }^{1}$ - Elisabeth H. Bos ${ }^{2} \cdot$ G. Ingrid J. G. Rours ${ }^{3}$. Pieter J. Hoekstra ${ }^{4} \cdot$ Eelko Hak $^{1,5}$. \\ Tjalling W. de Vries ${ }^{6}$
}

Received: 23 November 2018 / Accepted: 11 April 2019 / Published online: 24 April 2019

(c) The Author(s) 2019

\begin{abstract}
In a recent meta-analysis, we found that atopic diseases, like asthma and allergic rhinitis, occur more frequently prior to the onset of attention-deficit/hyperactivity disorder (ADHD). Our aim was to determine the temporal order of the association between daily fluctuations in atopic disease symptoms and in ADHD symptoms in individual participants. In this observational study among 21 participants, age 7-16 years, we performed a replicated time-series analysis of symptom fluctuations in asthma and/or allergic rhinitis and ADHD. Data were collected through parents who filled in a daily online questionnaire during up to 50 days. In each individual, we investigated the temporal order of fluctuations in atopic disease symptoms and ADHD symptoms using a vector autoregressive (VAR) model while using sleep problems and medication use as covariates. For 16 out of 21 participants, we constructed a VAR model. For a majority of the participants, significant associations were detected between atopic disease symptoms and ADHD symptoms. The results were heterogeneous; the direction, sign, and timing of the relationship between ADHD, atopy, sleep problems, and medication use varied between individuals. This study provides additional evidence that the symptom expression of atopy and ADHD are related. However, the connection between both diseases in children is found to be heterogeneous within our study population.
\end{abstract}

Keywords Attention-deficit/hyperactivity disorder · Atopy $\cdot$ Pediatrics $\cdot$ Daily diary study

Jurjen van der Schans

j.van.der.schans@rug.nl

1 PharmacoTherapy, Epidemiology and Economics, Groningen Research Institute of Pharmacy, University of Groningen, Antonius Deusinglaan 1, 9713 AV Groningen, The Netherlands

2 Department of Developmental Psychology, Faculty of Behavioural and Social Sciences, University of Groningen, Grote Kruisstraat 2/1, 9712 TS Groningen, The Netherlands

3 Medical Center for Quality of Life, Kinderplein, Metroplein 88, 3083 BB Rotterdam, The Netherlands

4 Department of Child and Adolescent Psychiatry, University Medical Center Groningen, University of Groningen, Hanzeplein 1, 9713 GZ Groningen, The Netherlands

5 Department of Epidemiology, University Medical Center Groningen, Hanzeplein 1, 9713 GZ Groningen, The Netherlands

6 Department of Pediatrics, Medical Center Leeuwarden, Henri Dunantweg 2, 8934 AD Leeuwarden, The Netherlands

\section{Introduction}

Attention-deficit/hyperactivity disorder (ADHD) is a common neurobehavioral disorder with onset of symptoms before the age of 12 years [1], characterized by symptoms of inattention and hyperactivity/impulsivity that often lead to social impairments. ADHD is a multifactorial disorder associated with both genetic and environmental factors, possibly including nutrition [2].

ADHD and atopic diseases co-occur more often than would be expected on the basis of chance [3]. Studies on the association between atopic diseases and ADHD have shown conflicting results in the strength of an association. However, almost all detected an increased risk in either the presence of an atopic disease in those with ADHD or vice versa an increased risk of the development of ADHD in those with an atopic disease [4]. Large cohorts with a few measurement waves are only informative to detect general patterns, and are not suitable for the detection of dynamic relationships between symptoms on a patient level [5]. 
In view of the frequent co-occurrence of ADHD and atopic diseases, it is important to investigate the possibility of an underlying common causal pathway. Daily fluctuations in symptoms are a hallmark of both atopic diseases and ADHD. The expression of symptoms in both atopy and ADHD is among others dependent on treatment efficiency, compliance to treatment, and external factors, which may cause these symptom fluctuations in both diseases. By studying these fluctuations over time within a series of individual ADHD cases and examining the dynamic association between atopic symptom fluctuations and ADHD symptom fluctuations, we aimed to determine the temporal order of the association between symptoms of atopic diseases and symptoms of ADHD.

\section{Methods}

\section{Study design}

We performed a replicated time-series analysis of asthma and allergic rhinitis symptom fluctuations and ADHD symptom fluctuations in individual participants. The time-series data were collected by means of an online diary study. For a period of 50 days, one of the parents was asked to fill out daily questionnaires; to be done at a specific time, preferably the end of the day between 8 and $9 \mathrm{pm}$. The questionnaire was applied as a mobile phone application and was used to assess the asthma and allergic rhinitis symptoms, and the ADHD symptoms. One and the same parent were asked to fill out the baseline questionnaire and the daily questionnaires to prevent reporting bias.

\section{Study population}

The study population consisted of 21 children in the age of 7-16 years in whom the diagnosis ADHD was established by a child psychiatrist, a child psychologist, or a pediatrician. Asthma or allergic rhinitis was diagnosed by a physician. Children were excluded if they were diagnosed with any chronic disease other than atopic diseases or ADHD. Parents could not participate if they were not fluent in the Dutch language or were, otherwise, unable to fulfill the study procedures or had no access to the Internet. Children and their parents were recruited by their pharmacists through the IADB.nl prescription database and the patient database of a medical center in Rotterdam. Participants were asked to continue care as usual throughout the study. Our local medical ethical committee (METC UMCG) had waived the ethical approval for this observational study design according to the declaration of Helsinki. All participants were asked to fill in an informed consent form before participation of the study.

\section{Measures}

At baseline, for each participant, age, sex, and the ratings of the ADHD symptoms [26-item scale Swanson, Nolan, and Pelham Questionnaire (SNAP-26)], atopy [Control of Allergic Rhinitis and Asthma Test (CARAT)], and sleep problems [Children's Sleep Habits Questionnaire (CSHQ)] as rated by one of the parents in the week before the initiation of the study, were determined to describe the sample characteristics.

To assess the severity of ADHD symptoms during the diary period, we used the SNAP IV questionnaire, which is a frequently used, validated tool in ADHD studies that is based on the 18 DSM-IV ADHD items [6, 7]. The combined score of inattention and hyperactivity/impulsivity subscale scores was used as rating of ADHD symptoms (18 questions; total score range, 0-54). To assess the asthma and allergic rhinitis symptom severity the adjusted CARAT questionnaire was used (7 questions; total score range, 0-21). The CARAT questionnaire is a validated and consistent way to measure asthma and allergic rhinitis symptoms [8]. Currently, the Adapt Asthma application, which is based on the CARAT, is already in use to monitor asthma and rhinitis symptoms [9]. The questions and answer options of the SNAP and the CARAT questionnaire were adapted for daily measurement by changing the phrasing and time periods of the original questions were referring to. In total, parents needed to answer seven questions for atopy and eighteen questions for ADHD daily, with different response categories per question.

\section{Covariates}

Co-variables like age, gender, sleep problems, and medication use were determined during inclusion and as part of the daily measurement. To address the possible involvement of sleep problems, as suggested by the previous research into atopic eczema and ADHD [10,11], in the fluctuations in ADHD symptoms and atopic symptoms, we added eight questions concerning assessment of daytime sleepiness selected from the CSHQ questionnaire (8 questions; total score range, 7-31) to the daily diary. Higher scores reflect worse sleep. The questions and answer options of the CSHQ questionnaire were adapted for daily measurement during daytime by changing the phrasing and time periods the original questions were referring to. In addition, items on medication use of both atopic and ADHD medication were added to the daily diary ('How often did your child take a higher dosage of its regular medication due to allergic rhinitis and/or asthma?'; 'How often did your child take a higher dosage of its regular 
medication due to ADHD symptoms?'; total score range, $0-3)$ to account for the possible effects of fluctuations in medication use on symptom expression in the models. Sleep problems and medication use were added as a covariate instead of a confounder, because of the association reported between ADHD and atopic diseases independent of sleep and medication [4].

\section{Statistical analyses}

A vector autoregressive (VAR) model was used to investigate the dynamic associations between changes in symptoms in each individual participant as well as the possible involvement of covariates in this association [12]. An important feature of the VAR model is the possibility to examine the temporal dynamics between multiple time-series, which allows studying the temporal order of the association between atopy and ADHD symptoms accounting for potential bidirectional effects and effects of time-varying covariates like sleep problems and medication use $[12,13]$. Another important feature of the VAR technique is the ability to separate the dynamic longitudinal part from the simultaneous part of the associations between the variables and to correct for potential feedback effects [12].

A VAR model consists of a set of regression equations, in which each of the variables is regressed on its own lagged values (autocorrelation) as well as the lagged values of the other variables (cross-lagged associations) [19]. We build VAR models with two endogenous variables: atopy symptoms and ADHD symptoms. The variable sleep problems were added to the primary model as an extra endogenous variable, because sleep can influence and can be influenced by atopy and ADHD symptoms. Medication use was added to the model as an endogenous variable if there was variation in medication use during the follow-up period. We first investigated the optimal lag number to be used in each of the models (a lag $=1$ day), according to a comparison of the final prediction error of each lag model. Since VAR models assume stationarity, we subsequently compared four different types of VAR models (constant, trend, both, and none) to determine if an additional trend or intercept term was needed in the model. Finally, we selected the optimal model with the Akaike Information Criterion (AIC) based on the smallest value of AIC, a measure of relative quality of the fitted model.

To assess the temporal order of the association between atopy and ADHD symptoms, and the possible involvement of sleep problems therein, we determined the independent cross-lagged associations between each variable. In addition, we assessed the contemporaneous correlations between symptoms of atopy, ADHD, and sleep problems per participant to determine the simultaneous associations between (or co-occurrence of) the expression of symptoms. These can be computed from the residuals of the VAR model [19]. A $p$ value $\leq 0.05$ was considered to be statistically significant. Per individual model, multiple diagnostic tests were performed to test for stability and homoscedasticity of the model [14]. For six out of the participants, variables were $\log$-transformed for either the score of atopy (participants $10,11,13$, and 16) or for both atopy and sleep (participants 2 and 3), because the score itself is not normally distributed as observed by the histogram. Models were fitted in $\mathrm{R}$ (R Foundation for Statistical Computing, software version 3.4.0, Vienna, Austria) using the package vars. $p$ values $<0.05$ were considered to denote statistically significant associations and $p$ values $<0.10$ were considered to denote the trend of associations.

Simulation studies have shown that, for VAR modeling, a minimum of 30 time points is needed, although larger numbers of observations yield more reliable results [14]. We chose for a time-series length of 50 measurement points taking into account the possibility of missing data. Missing values on the daily sum scores of ADHD, allergy, and sleep were dealt with using multiple imputations on the individual level [15]. Considering the percentage of the missing measurements per participant, we obtained five imputed data sets for participants $1,2,3,4,9,10,11,13$, and 16 , ten imputed data sets for participants $5,7,15,17,18$, and 21 , and 15 imputed data sets for participants 19 . The imputed disease ratings were then computed by taking the average scores generated in each imputation. Subsequently, ADHD ratings were calculated by summing up the separate inattentive score and hyperactive score.

\section{Results}

Five of the twenty-one participants (\#6, \#8, \#12, \#14, and \#20) were excluded from analyses, because not enough observations $(n<30)$ were completed during the data collection. A description of the characteristics of our study sample is presented in Table 1. All participants were between 7 and 16 years of age at the start of the study period. The majority (61.9\%) were male. Per participant, the baseline ratings of ADHD symptoms (SNAP-26), atopy (CARAT), and sleep (CSHQ) are shown. Within our study sample, nine out of the twenty-one participants (42.9\%) were diagnosed with both asthma and allergic rhinitis.

\section{Temporal order of the relationship between atopy, ADHD, and sleep problems}

In all models, an optimal lag of 1 day was selected to be optimal. Both the temporal direction as well as the sign (positive or negative) and size of the effect varied between the participants. Five out of the 16 participants showed a 
Table 1 Characteristics of the study group $(n=21)$

\begin{tabular}{|c|c|c|c|c|c|c|c|c|c|c|c|c|c|c|c|c|c|c|c|c|c|c|}
\hline \multirow[t]{2}{*}{ Characteristics } & \multirow[t]{2}{*}{ Mean (SD) } & \multicolumn{21}{|c|}{ Participants } \\
\hline & & 1 & 2 & 3 & 4 & 5 & 6 & 7 & 8 & 9 & 10 & 11 & 12 & 13 & 14 & 15 & 16 & 17 & 18 & 19 & 20 & 21 \\
\hline Completed observations & & 39 & 48 & 46 & 49 & 30 & 5 & 30 & 22 & 43 & 44 & 42 & 21 & 50 & 21 & 37 & 43 & 32 & 40 & 32 & 4 & 42 \\
\hline Gender (female), $n$ & $8(38.1 \%)$ & 우 & $\hat{0}$ & $\hat{0}$ & $\hat{0}$ & $\hat{\sigma}$ & $0^{\pi}$ & 우 & 우 & $\hat{0}$ & $\hat{0}$ & $\hat{0}$ & 우 & $\hat{0}$ & $\hat{0}$ & 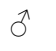 & $\hat{0}$ & 우 & 우 & $\hat{0}$ & q & 우 \\
\hline Age & $11.3(2.3)$ & 12 & 11 & 12 & 7 & 11 & 16 & 13 & 10 & 9 & 12 & 8 & 12 & 11 & 12 & 11 & 15 & 10 & 14 & 13 & 12 & 7 \\
\hline ADHD diagnosis & & + & + & + & + & + & + & + & + & + & + & + & + & + & + & + & + & + & + & + & + & + \\
\hline Asthma diagnosis & & + & - & - & + & + & + & - & - & + & - & + & + & - & - & + & + & + & - & - & - & + \\
\hline Allergic rhinitis diagnosis & & + & + & + & + & + & + & + & + & + & + & + & + & + & + & - & + & - & + & + & + & + \\
\hline \multicolumn{23}{|l|}{ SNAP-26 score } \\
\hline Total & $34.6(10.6)$ & 60 & 37 & 12 & 40 & 35 & 39 & 31 & 32 & 17 & 18 & 39 & 34 & 30 & 48 & 36 & 40 & 31 & 41 & 43 & 36 & 27 \\
\hline Inattention & $18.7(4.7)$ & 19 & 17 & 11 & 18 & 17 & 23 & 20 & 15 & 9 & 17 & 21 & 17 & 14 & 27 & 14 & 20 & 27 & 19 & 26 & 21 & 20 \\
\hline Hyperactivity/impulsivity & $11.0(5.7)$ & 18 & 11 & 1 & 20 & 15 & 8 & 9 & 15 & 7 & 0 & 14 & 17 & 13 & 18 & 16 & 11 & 1 & 10 & 9 & 12 & 7 \\
\hline CARAT score & $10.1(5.6)$ & 7 & 6 & 7 & 12 & 17 & 4 & 11 & 4 & 8 & 8 & 7 & 10 & 12 & 28 & 15 & 11 & 17 & 11 & 8 & 6 & 4 \\
\hline CSHQ score & $47.8(8.1)$ & 60 & 52 & 42 & 45 & 53 & 54 & 56 & 37 & 37 & 38 & 37 & 49 & 51 & 68 & 47 & 53 & 40 & 46 & 48 & 45 & 45 \\
\hline Overall sleep grade $(0-10)$ & $6.8(1.8)$ & 4 & 8 & 8 & 7 & 5 & 3 & 5 & 8 & 9 & 8 & 9 & 6 & 7 & 5 & 4 & 8 & 9 & 7 & 7 & 7 & 8 \\
\hline
\end{tabular}

$S D$ standard deviation, $n$ sample size, $A D H D$ attention-deficit/hyperactivity disorder, SNAP Swanson Nolan and Pelham questionnaire, CARAT control of allergic rhinitis and asthma test, $C S H Q$ children's sleep habits questionnaire

q girl, $\widehat{\partial}$ boy, + yes, - no

Table 2 Significant and trend 1-day cross-lagged associations

\begin{tabular}{|c|c|c|c|c|c|c|c|c|c|c|c|c|}
\hline \multirow[t]{2}{*}{ Participant ID } & \multicolumn{2}{|c|}{ Atopy $\rightarrow$ ADHD } & \multicolumn{2}{|c|}{$\mathrm{ADHD} \rightarrow$ atopy } & \multicolumn{2}{|c|}{ Sleep $\rightarrow$ ADHD } & \multicolumn{2}{|c|}{$\mathrm{ADHD} \rightarrow$ sleep } & \multicolumn{2}{|c|}{ Sleep $\rightarrow$ atopy } & \multicolumn{2}{|c|}{ Atopy $\rightarrow$ sleep } \\
\hline & $B$ & $p$ value & $B$ & $p$ value & $B$ & $p$ value & $B$ & $p$ value & B & $p$ value & $B$ & $p$ value \\
\hline 1 & 3.59 & 0.004 & & & -1.51 & 0.013 & & & & & 0.71 & 0.023 \\
\hline $2^{\mathrm{a}}$ & & & & & -9.27 & 0.075 & & & & & & \\
\hline \multicolumn{13}{|l|}{$3^{a}$} \\
\hline 4 & & & -0.13 & 0.056 & & & & & & & & \\
\hline \multicolumn{13}{|l|}{5} \\
\hline \multicolumn{13}{|l|}{7} \\
\hline 9 & 0.40 & 0.052 & 0.22 & 0.051 & & & & & & & & \\
\hline $10^{\mathrm{a}}$ & & & 0.12 & 0.007 & & & & & & & & \\
\hline $11^{\mathrm{a}}$ & -1.25 & 0.048 & & & & & & & -0.07 & 0.058 & & \\
\hline \multicolumn{13}{|l|}{$13^{\mathrm{a}}$} \\
\hline 15 & & & 0.079 & 0.096 & & & 0.13 & 0.045 & & & 0.31 & 0.084 \\
\hline \multicolumn{13}{|l|}{16} \\
\hline \multicolumn{13}{|l|}{17} \\
\hline $18^{\mathrm{a}}$ & & & & & & & & & 0.33 & 0.032 & -0.48 & 0.004 \\
\hline 19 & & & & & & & 0.30 & 0.097 & & & & \\
\hline $21^{\mathrm{a}}$ & -0.42 & 0.071 & & & & & & & -0.35 & 0.052 & & \\
\hline
\end{tabular}

$\rightarrow$ dynamic effect, atopy sum score of atopic symptoms, ADHD sum score of attention-deficit/hyperactivity disorder symptoms, sleep sum score of sleep problems, $B$ unstandardized regression coefficient

${ }^{a}$ Models are adjusted for ADHD medication use (participants 2, 3, 10, 11, 13, and 18) and allergic medication use (participants 10, 13, and 21)

significant $(p<0.05)$ cross-lagged association between some of the variables (Table 2). In participant 1 , an increase in expression of atopic symptoms preceded a worsening in ADHD symptoms $(B=3.59 ; p=0.004)$ and sleep problems $(B=0.71 ; p=0.023)$, while increases in sleep problems were followed by decreases in ADHD symptoms $(B=-1.51$; $p=0.013)$. In participant 10, an increase in ADHD symptoms was followed by an increase in symptoms of atopy $(B=0.12 ; p=0.007)$. In contrast to the other participants, in participant 11 , a negative association was detected in which a decrease in atopic symptoms $(B=-1.25 ; p=0.048)$ was followed by an increase in ADHD symptoms. Participant 
15 showed a worsening of sleep, preceded by an increase in ADHD symptoms $(B=0.13 ; p=0.045)$. In participant 18 , sleep problems and atopic symptoms were associated in both directions, i.e., an increase in sleep problems preceded an increase in atopic symptoms $(B=0.33 ; p=0.032)$, while an increase in atopic symptoms preceded a decrease in sleep problems $(B=-0.48 ; p=0.004)$.

\section{Contemporaneous associations}

In addition to the dynamic (cross-lagged) associations between symptoms of atopy, ADHD, and sleep problems, we also addressed the co-occurrence of these symptoms. Table 3 shows an overview of the contemporaneous expression of symptoms of atopy, ADHD, and sleep problems. Atopy and ADHD, sleep problems and ADHD, and sleep problems and atopy were contemporaneously associated with a number of participants. In 4 out of 16 participants, the contemporaneous association between atopy and ADHD was significant. The sign of this association was positive in 3 out of 4 of these participants. Also most of the contemporaneous associations between sleep problems and atopy or ADHD symptoms had a positive sign.

\section{Associations between medication use and the symptom variables/sleep problems}

In seven participants, the medication use related to ADHD (participants 2, 3, 10, 11, 13, and 18) and to allergy (participants 10, 13, and 21) varied during the study period. For this reason, these variables were included in the VAR models of these participants as an additional endogenous parameter. A number of cross-lagged associations between medication use and the other variables were present: Participant 10 showed a significant crosslagged association between sleep problems $(B=0.14$; $p=0.048$ ) and ADHD medication: an increase in sleep problems was followed by an increase in ADHD medication. In participant 21, significant cross-lagged associations between allergic-related medication on one hand, and ADHD $(B=4.98 ; p=0.009)$, sleep problems $(B=-0.04$; $p=0.012)$, and atopy $(B=0.06 ; p=0.006)$ on the other hand were detected.

When evaluating the contemporaneous associations between medication use and the symptom expression of the different diseases, we detected a negative association between ADHD medication and atopy $(r=-0.29$; $p=0.042$ ) in participant 2 and a negative association between ADHD medication and sleep problems $(r=-0.36 ; p=0.010)$ in participant 2 and $(r=-0.29$; $p=0.044$ ) participant 10. Furthermore, we observed a positive association between allergic medication and ADHD ( $r=0.34 ; p=0.013$ ) and between allergic medication and atopy $(r=0.66 ; p<0.001)$ in participant 21 .

In participants $3,11,13$, and 18 , no association was observed between medication use and either sleep, ADHD, and/or atopy.
Table 3 Contemporaneous significant and trend associations between symptoms of atopy, ADHD, and sleep problems

\begin{tabular}{|c|c|c|c|c|c|c|}
\hline \multirow[t]{2}{*}{ Participant ID } & \multicolumn{2}{|c|}{ Atopy and ADHD } & \multicolumn{2}{|c|}{ Sleep and ADHD } & \multicolumn{2}{|c|}{ Sleep and atopy } \\
\hline & $r$ & $p$ value & $r$ & $p$ value & $r$ & $p$ value \\
\hline \multicolumn{7}{|l|}{1} \\
\hline $2^{\mathrm{a}}$ & -0.29 & 0.093 & -0.24 & 0.045 & 0.40 & 0.004 \\
\hline \multicolumn{7}{|l|}{$3^{\mathrm{a}}$} \\
\hline 4 & & & 0.26 & 0.067 & & \\
\hline 5 & & & & & -0.70 & $<0.001$ \\
\hline 7 & 0.28 & 0.080 & & & 0.27 & 0.093 \\
\hline \multicolumn{7}{|l|}{9} \\
\hline $10^{\mathrm{a}}$ & 0.31 & 0.032 & -0.45 & 0.001 & & \\
\hline \multicolumn{7}{|l|}{$11^{\mathrm{a}}$} \\
\hline \multicolumn{7}{|l|}{$13^{\mathrm{a}}$} \\
\hline \multicolumn{7}{|l|}{15} \\
\hline 16 & 0.30 & 0.040 & 0.25 & 0.089 & & \\
\hline 17 & -0.43 & 0.003 & & & & \\
\hline $18^{\mathrm{a}}$ & 0.27 & 0.056 & & & & \\
\hline \multicolumn{7}{|l|}{19} \\
\hline $21^{\mathrm{a}}$ & 0.66 & $<0.001$ & 0.34 & 0.014 & 0.50 & $<0.001$ \\
\hline
\end{tabular}

$A D H D$ attention-deficit/hyperactivity disorder, $r$ correlation coefficient

${ }^{a}$ Models are adjusted for ADHD medication use (participants 2, 3, 10, 11, 13, and 18) and allergic medication use (participants 10, 13, and 21) 


\section{Differentiating inattention and hyperactivity/ impulsivity}

When stratifying the ADHD sum score into separate scores of inattention and hyperactivity/impulsivity, similar results were observed. A notable difference was that the crosslagged association between sleep problems and ADHD symptoms in participant 2 appeared to be mainly accounted for by hyperactivity/impulsivity symptoms. The same was true for the cross-lagged association between ADHD and atopic symptoms in participant 4 and between ADHD and sleep problems in participant 15 , which were also accounted for by hyperactivity/impulsivity symptoms. The opposite was true for the cross-lagged association between ADHD symptoms and atopic symptoms in participant 10 , which was accounted for by symptoms of inattention.

A separate cross-lagged association appeared in participant 3 for between inattention and sleep problems $(B=0.02$; $p=0.072)$ and in participant 16 between inattention and atopic symptoms $(B=0.07 ; p=0.031)$.

As regards the contemporaneous associations between the different symptoms, also similar results were observed as in the original analyses. The contemporaneous association between atopy and ADHD, and sleep problems and ADHD in participant 10, atopy and ADHD in participant 16, and sleep problems and ADHD in participant 21 were accounted for by symptoms of inattention. In comparison with Table 3 , new significant associations were found between atopy and inattention $(r=0.40 ; p=0.005)$, and sleep problems and inattention $(r=0.37 ; p=0.010)$ in participant 4 , sleep problems and inattention $(r=0.46 ; p=0.002)$ in participant 7 , and sleep problems and hyperactivity/impulsivity $(r=-0.37 ; p=0.010)$ in participant 9 .

\section{Discussion}

To our knowledge, this is the first study using time-series analysis to examine the temporal order of an association between atopic disease symptoms and ADHD symptoms. For a majority of the participants, significant associations were detected between atopic disease symptoms and ADHD symptoms. However, the results were heterogeneous, with varying direction, sign, and timing of the relationship between ADHD, atopy, sleep problems, and medication use between participants. In other words, the observed associations were not consistent across the participants. Regarding ADHD symptoms as outcome, our analysis showed a relationship between symptoms of allergy or sleep problems and ADHD symptoms in some of the participants. There was also negative cross-lagged association present between sleep problems and ADHD. The cross-lagged association between atopy and ADHD differed in direction between the participants. Regarding atopic symptoms as the outcome, only one participant showed a significant cross-lagged association between ADHD and allergy symptoms. When assessing the more direct contemporaneous relationships, our analyses showed an association in many of the participants between ADHD symptoms (inattention, hyperactivity/ impulsivity, or combined) or ADHD medication use, allergy, and/or sleep problems. The associations differed in both sign and strength.

Based on different group-based observational studies, the association between atopy and ADHD is considered weak-to-moderate $[3,16]$. Although the association between symptom patterns in some participants does correspond with these between-subject results, in other participants, the relationship between ADHD and atopy was absent or even reversed from what would be expected based on the literature of between-subject results. This might be explained by the fact that both atopic disease and ADHD are complex and heterogeneous diseases with regards to etiology, symptom expression, and treatment $[17,18]$. As a second reason for the heterogeneity of our results, proxy reporting by parents may be less suitable for assessing sleep problems and symptoms of allergy. Another reason may be that our series were rather short, which has limited our power. Future studies should preferably collect longer series. In general, the heterogeneity of our study results may explain why the effect size of the association between atopy and ADHD in group-based studies is often weak. In a heterogeneous population, in which the association between atopy and ADHD is not present in every individual, the group-based association between the two diseases can be diluted, while the association between the diseases can still be significant at an individual level. This is illustrated by our results in which the cross-lagged association between atopy and subsequent ADHD symptoms is reversed in participant 1 compared to participant 11, while both show a significant association between the symptoms. A similar pattern is evident when comparing the between-subjects correlation and the withinsubject correlation, representing the correlation over individuals and over time, respectively. Participants 5 and 7 both scored relatively high on the baseline score of atopy and sleep problems compared to the other participants, while at an individual level over time atopy and sleep problems showed a negative contemporaneous correlation in participant 5 and a positive contemporaneous correlation in participant 7 .

\section{Clinical relevance}

A recent study showed that treatment of allergic rhinitis decreased ADHD symptom severity significantly compared with healthy controls and children with pure ADHD [19]. In our study, in only one participant, increases in allergic 
symptoms were followed by subsequent increases in ADHD symptoms, suggesting that a specific treatment of the atopic symptoms might, perhaps, improve the symptoms of ADHD in this individual. On the contrary, treatment of ADHD might be beneficial for the symptoms of allergy of participant 10 and participant 16, because increases in ADHD symptoms were followed by increases in atopic symptoms in these participants. Participants 4 (inattention), 10, 16, and 21 showed a contemporaneous positive association between allergic and ADHD symptoms, in which an improvement of atopic symptoms could potentially benefit the participant by also improving the ADHD symptom expression, and vice versa. Because of a direct association between ADHD symptoms and sleep problems in participants 3 (inattention) and 15 , and a contemporaneous positive association between ADHD and sleep problems in participants 4 (inattention), 7 (inattention), and 21, these participants might benefit from improving their sleep for amelioration of their ADHD symptoms. Adding to the complexity of the different associations between ADHD, atopy, sleep problems, and the subsequent possible clinical implications is the use of medication. In, for example, participant 21, lowering of allergic medication could possibly benefit the participant in her ADHD symptoms. However, a direct positive association still exists between symptoms of allergy and ADHD when controlling for medication use, which would suggest a contradictory clinical implication of an increase in treatment of allergy and/or ADHD to improve symptoms of both disorders.

Taking into account the group-based cross-sectional and prospective association between atopy and ADHD in the literature [3], it might be expected that this study would have shown a more consistent direct or lagged effect between ADHD and atopy. Because no such consistent relationship was established in this study, it is possible that the association between atopy and ADHD partly exists because of an indirect mechanism, like genetic predisposition, or that the temporal association of cause and effect is stretched over a longer period of time. Multiple pathophysiological hypotheses have been raised to explain the possible comorbidity between atopic diseases and ADHD [20]. Among others, chronic stress and chronic inflammation have been raised as possible connections between atopy and ADHD. Because of the limited study period, it was not possible to measure these possible effects. While a general group-based approach of treating atopy to improve ADHD symptoms, and vice versa, will not necessarily benefit the whole group, individualized assessment of interdisciplinary treatment of both diseases could benefit individual participants.

\section{Strengths and limitations}

Our findings should be interpreted in the light of the strengths and limitations. Examining symptom fluctuations in allergy, ADHD, and sleep problems on a daily basis allowed us to observe the concurrent as well as the temporal relationships between the different variables. A limitation was the small sample size of the study, which limits the generalizability of the results. However, due to the multiple repeated measurement points per participant, it was possible to examine each individual participant in detail and to explore the possible temporal order of the association between atopy and ADHD. Combining both study types, with repeated measurements of symptom expression of both atopy and ADHD in a large cohort, would benefit the study of the association between both diseases.

Another limitation has been that the participants were followed daily for a maximum of 50 days, but the effects of the symptom expression of one of the diseases could accumulate over time to have a larger and more significant effect on the long term [21, 22]. For example, weekly measurements over a longer period of time could reveal a more consistent association between the symptom expressions of both diseases. In addition, adding atopic eczema, food allergies, and a more comprehensive assessment of sleep problems would be recommendable for future studies. The role of age of onset and disease duration in examining the association between symptoms of ADHD, atopy, and sleep problems may also be considered in future studies. We did not add more variables to our daily questionnaire to avoid loss to follow-up of our participants.

Time-series analysis is a potentially useful method to assess the temporal order of the association between atopy and ADHD. This study provides additional evidence that the symptom expression of atopy and ADHD is related. However, the connection between symptom expressions of both diseases in children is heterogeneous, and differs in sign, direction, strength, and timing in our study population. To enable improvement in the care of patients, personalized treatment needs of children with both atopic disease and ADHD should be assessed at an individual basis.

\section{Compliance with ethical standards}

Conflict of interest The authors declare that they have no conflict of interest.

Open Access This article is distributed under the terms of the Creative Commons Attribution 4.0 International License (http://creativecommons.org/licenses/by/4.0/), which permits unrestricted use, distribution, and reproduction in any medium, provided you give appropriate credit to the original author(s) and the source, provide a link to the Creative Commons license, and indicate if changes were made. 


\section{References}

1. Centers for Disease Control and Prevention (CDC) (2010) Increasing prevalence of parent-reported attention-deficit/hyperactivity disorder among children-united states, 2003 and 2007. MMWR 59(44):1439-1443

2. Feldman HM, Reiff MI (2014) Clinical practice: attention deficithyperactivity disorder in children and adolescents. N Engl J Med 370(9):838-846

3. Schans JV, Cicek R, de Vries TW, Hak E, Hoekstra PJ (2017) Association of atopic diseases and attention-deficit/hyperactivity disorder: a systematic review and meta-analyses. Neurosci Biobehav Rev 74(Pt A):139-148

4. Schmitt J, Buske-Kirschbaum A, Roessner V (2010) Is atopic disease a risk factor for attention-deficit/hyperactivity disorder? A systematic review. Allergy 65(12):1506-1524

5. Hamaker E (2012) Why researchers should think 'within-person': a paradigmatic rationale. In: Handbook of research methods for studying daily life. Guilford Press, New York, pp 43-61

6. The MTA Cooperative Group (1999) A 14-month randomized clinical trial of treatment strategies for attention-deficit/hyperactivity disorder. Arch Gen Psychiatry 56(12):1073-1086

7. Jensen P, Arnold LE, Richters JE, Severe JB, Vereen D, Vitiello B, Schiller E, Hinshaw S, Elliott GR, Conners CK, Wells KC (1999) Moderators and mediators of treatment response for children with attention-deficit/hyperactivity disorder: the multimodal treatment study of children with attention-deficit/hyperactivity disorder. Arch Gen Psychiatry 56(12):1088-1096

8. Fonseca JA, Nogueira-Silva L, Morais-Almeida M et al (2010) Validation of a questionnaire (CARAT10) to assess rhinitis and asthma in patients with asthma. Allergy 65(8):1042-1048

9. Kosse RC, Bouvy ML, de Vries TW et al (2017) mHealth intervention to support asthma self-management in adolescents: the ADAPT study. Patient Prefer Adher 11:571-577

10. Romanos M, Gerlach M, Warnke A, Schmitt J (2010) Association of attention-deficit/hyperactivity disorder and atopic eczema modified by sleep disturbance in a large population-based sample. J Epidemiol Community Health 64(3):269-273

11. Schmitt J, Chen CM, Apfelbacher $C$ et al (2011) Infant eczema, infant sleeping problems, and mental health at 10 years of age: the prospective birth cohort study LISAplus. Allergy 66(3):404-411
12. Brandt PT, Williams JT (2007) Multiple time series models. Vol 07-148. Sage Publications, Thousand Oaks, p 99

13. Rosmalen JG, Wenting AM, Roest AM, de Jonge P, Bos EH (2012) Revealing causal heterogeneity using time series analysis of ambulatory assessments: application to the association between depression and physical activity after myocardial infarction. Psychosom Med 74(4):377-386

14. Lütkepohl $\mathrm{H}$ (2005) New introduction to multiple time series analysis. Springer, Berlin, p 764. http://www.loc.gov/catdir/enhan cements/fy0663/2005927322-d.html

15. Rubin DB (1996) Multiple imputation after 18+ years. J Am Stat Assoc 91(434):473-489

16. Miyazaki C, Koyama M, Ota E et al (2017) Allergic diseases in children with attention deficit hyperactivity disorder: a systematic review and meta-analysis. BMC Psychiatry 17(1):120-017-1281-7

17. Kendler KS, Zachar P, Craver C (2011) What kinds of things are psychiatric disorders? Psychol Med 41(6):1143-1150

18. Garden FL, Simpson JM, Marks GB, CAPS Investigators (2013) Atopy phenotypes in the childhood asthma prevention study (CAPS) cohort and the relationship with allergic disease: Clinical mechanisms in allergic disease. Clin Exp Allergy 43(6):633-641

19. Yang MT, Chen CC, Lee WT, Liang JS, Fu WM, Yang YH (2016) Attention-deficit/hyperactivity disorder-related symptoms improved with allergic rhinitis treatment in children. Am J Rhinol Allergy 30(3):209-214

20. Buske-Kirschbaum A, Schmitt J, Plessow F, Romanos M, Weidinger S, Roessner V (2013) Psychoendocrine and psychoneuroimmunological mechanisms in the comorbidity of atopic eczema and attention deficit/hyperactivity disorder. Psychoneuroendocrinology 38(1):12-23

21. Wichers M, Peeters F, Rutten BP et al (2012) A time-lagged momentary assessment study on daily life physical activity and affect. Health Psychol 31(2):135-144

22. Stavrakakis N, Booij SH, Roest AM, de Jonge P, Oldehinkel AJ, Bos EH (2015) Temporal dynamics of physical activity and affect in depressed and nondepressed individuals. Health Psychol 34S: $1268-1277$ 